\title{
Effect of Gramicidin D on the Compressibility and Volume Fluctuations of DPPC - Peptide Bilayers: A Densitometry and Sound Velocimetry Study
}

\author{
Linus N. Okoro \\ American University of Nigeria \\ Yola, Nigeria \\ Tel: 234-805-604-8688Ｅ-mail: linus.okoro@aun.edu.ng
}

\begin{abstract}
The effects of increasing gramicidin $\mathrm{D}(\mathrm{gD})$ concentration on the partial specific volume, $v^{\circ}$, and the adiabatic compressibility coefficient of the lipid, $\beta_{S}^{\text {lipid }}$ of the bilayer reveals a continual decrease in $v^{\circ}$ and $\beta_{S}^{\text {lipid }}$ (within the range of concentration studied) with concentration, except between 5 and $7.5 \%$, where a very slight decrease was observed. At $\mathrm{gD}$ concentrations higher than $5 \mathrm{~mol} \%$, the isothermal compressibility coefficient, $\beta_{T}^{\text {lipid }}$ is greater than $\beta_{S}{ }^{\text {lidid }}$ at the gel-fluid region by $\sim 10-20 \%$ in the entire temperature range covered. The maximum value of the relative volume fluctuation of $12 \%$ is reached for DPPC at the main transition, and is strongly dampened upon addition of $\mathrm{gD}$. A gradual decrease in the calculated relative volume fluctuations with $\mathrm{gD}$ concentration at $T_{\mathrm{m}}$ is observed between concentrations 1 and $10 \mathrm{~mol} \%$.
\end{abstract}

Keywords: Gramicidin D (gD), Phospholipid, DPPC, Pressure perturbation calorimetry, Ultrasound velocimetry, Densitometry, Fluctuations

\section{Introduction}

In 1939 the French-American microbiologist Rene Dubos isolated the substance tyrothricin and later showed that it was composed of two substances, gramicidin $(20 \%)$ and tyrocidine $(80 \%)$. These were the first antibiotics to be manufactured commercially. Gramicidin is a heterogeneous mixture of antibiotic compounds, Gramicidins A, B and C, making up $80 \%, 6 \%$, and $14 \%$ respectively [Bourinbaiar 1997], all of which are obtained from the soil bacterial species Bacillus brevis and called collectively Gramicidin D. Gramicidin D are linear pentadecapeptides; that is chains made up of 15 amino acids [Burkhart, 1999]. This is in contrast to gramicidin S, which has a cyclic peptide chain. Gramicidin is active against Gram-positive bacteria, except for the Gram-positive bacilli, and against selected Gram-negative organisms, such as Neisseria bacteria.

Gramicidin has the general formula: formyl-L-X-Gly-L-Ala-D-Leu-L-Ala-D-Val-L-Val-D-Val-L-Trp-D-Leu-L-Y-D-Leu-L-Trp-D-Leu-L-Trp-ethanola mine. $\mathrm{X}$ and $\mathrm{Y}$ depend upon the gramicidin molecule. There exists valine and isoleucine variants of all three gramicidin species and ' $\mathrm{X}$ ' can be either valine or isoleucine. $\mathrm{Y}$ determines which is which; in the place of $\mathrm{Y}$, Gramicidin A contains Tryptophan, B contains Phenylalanine and C contains Tyrosine. The alternating stereochemical configurations (in the form of $\mathrm{D}$ and $\mathrm{L}$ ) of the amino acids is vital to the formation of the $\beta$-helix. The chain assembles inside of the hydrophobic interior of the cellular lipid bilayer to form a $\beta$-helix. The helix itself is not long enough to span the membrane but it dimerises to form the elongated channel needed to span the whole membrane.

\subsection{Lipid - Gramicidin Interaction}

The primary interest of gramicidin lies in its ability to form ion channels in lipid membranes. Gramicidin has been reported to form specific channels across the cell membrane and to enhance the transport of cations [Woolf 1994]. Gramicidin has no charged or hydrophilic side chains, and its aqeous solubility is low. As both the amino and carboxy termini of the molecule is blocked, gramicidin has been found to partition strongly into the hydrophobic region of phospholipid membranes and to maintain the liquid - crystalline state [Short 1987, Glowka 2005]. Extensive studies have been made on the influence of gramicidin on lipid polymorphism and of the ordering effects on the lipid chains [Killian 1992, Rice 1979, Kharakoz 1993, Szule 2003]. Gramicidin S has been found to progressively decrease the phase transition of DMPC vesicles as well as to decrease the degree of cooperativity of the main phase transition and to increase the volume compressibility of the vesicles [Krivanek 2001, Lewis 1999]. 
The PFG-NMR method has been used in microscopically oriented bilayers to investigate the effect of the Gramicidin D on the lateral diffusion of DMPC [Orädd 2004]. No evidence of linear aggregate of gramicidin in the gel phase was found. Aggregation of gramicidin A in phospholipids has been reported [Ivanova 2003].

It has equally been observed that gramicidin insertion into the DMPC bilayer structure has significant influence on the lipid bilayer structure and temperature, pressure phase behaviour [Zein 2000, Eisenblätter 2006]. Further, the interaction between integral proteins and lipids has been found to depend on the relative length of their hydrophobic core, a concept known as the 'hydrophobic matching' (Woolf 1996). Gramicidin modulates the spontaneous curvature properties of the phospholipid assemblies [Szule 2003]. The effect of gramicidin D on the conductance and electroporation thresholds of planar bilayer membranes has been examined and was found to change their mechanical properties [Troiano 1999]. It has been shown as well, that the presence of peptide or proteins in the membrane can significantly affect the dynamic structure of the bulk lipid [Ge 1994].

The goal of this study is to incorporate different gramicidin D concentrations into DPPC bilayer, and to determine the effects of increasing concentrations of gramicidin D on the lipid bilayer membrane compressibility and volume fluctuations in their different transition phases. To this end, molecular acoustics (ultrasound velocimetry and densitometry) was utilized at varying significant gramicidin $\mathrm{D}$ concentrations. This is the first time the two methods could be employed simultaneously for the study of DPPC - gramicidin D interactions, to the best of my knowledge. This study has been able to reveal a considerable influence of gramicidin D on the mechanical, volume and compressibility properties of DPPC bilayer.

\section{Materials and methods}

\subsection{Sample Preparation}

1,2-Dipalmitoyl-3-sn-phosphatidylcholine (DPPC) was purchased from Avanti Polar Lipids (Alabaster, AL, USA), while Gramicidin D as a lyophilized solid was obtained from Sigma-Aldrich. Both were used without further purification. Multilamellar vesicles (MLV) of DPPC and melittin with designated mole ratios were mixed in chloroform-methanol mixture $(3: 1 \mathrm{v} / \mathrm{v})$ and dried as a thin film under a stream of nitrogen and then freeze-dried in a freeze-dryer (Christ, Osterode, Germany) under high vacuum overnight. The lipid films were hydrated in a Tris buffer ( $10 \mathrm{mM}$ Tris- $\mathrm{HCl}, 100 \mathrm{mM} \mathrm{NaCl}, \mathrm{pH} 7.4$ ), followed by vortexing at $\sim 60^{\circ} \mathrm{C}$ (above the main phase transition temperature, $T_{\mathrm{m}}$, of DPPC $\left(\sim 41.5^{\circ} \mathrm{C}\right.$ [Cevc 1987, Okoro 2008], and five freeze-thaw cycles, resulting in homogeneous multilamellar vesicles (MLVs). Large unilamellar vesicles (LUVs) of uniform shape and size used in the ultrasound velocity and the density measurements were prepared from the MLVs by extrusion [MacDonald 1991] using a Mini-Extruder (Avanti Polar Lipids Inc., Alabaster, AL, USA), and passing them through $100 \mathrm{~nm}$ Nuclepore ${ }^{\circledR}$ Polycarbonate Track-Etch ${ }^{\mathrm{TM}}$ Membranes (Whatman GmbH, Dassel, Germany) at $\sim 60^{\circ} \mathrm{C}$. The final DPPC concentration used in the ultrasound velocity and the density measurements was $5 \mathrm{mg} / \mathrm{mL}$.

\subsection{Ultrasound velocity and density measurements}

The ultrasound velocity $u$ of the vesicles was determined simultaneously using a differential ultrasonic resonator device ResoScan (TF Instruments, Heidelberg, Germany, operating in a frequency range of 7.2 - 8.5 MHz. [Eggers 1969, Eggers 1973, Stuehr 1965].

Ultrasonic measurements are extremely sensitive to temperature changes. Reproducibility and accuracy is therefore crucially dependent on TFI's Ultra-high Precision Peltier-Thermostat that reaches temperature constancy better than $1 \mathrm{mK}\left(0,001{ }^{\circ} \mathrm{C}\right)$, and features a fast heating, cooling and equilibration time between 5 and $85^{\circ} \mathrm{C}$.

The sound velocity of the lipid dispersion was determined relative to that in the buffer solution at the same temperature in terms of the velocity number, $[u]$, defined as [Stuehr 1965].

$$
[u]=\left(u-u_{0}\right) / u_{0} c
$$

Where $u$ and $u_{\mathrm{o}}$ denote the sound velocity in the solution and in the solvent, respectively, and $c$ is the solute concentration in $\mathrm{mol} / \mathrm{L}$.

The densities, $\rho$ and $\rho_{0}$, of the lipid solution and the solvent, respectively were measured by a high-precision density meter DMA 5000 (Anton Paar, Graz, Austria) based on the mechanical oscillator principle [Kratky 1973], corrected for viscosity-induced errors.

The partial molar volume, $V^{0}$, of the lipid is evaluated from the density data by the given relation:

$$
V^{\circ}=\left(\frac{\partial V}{\partial n}\right) \cong \frac{M}{\rho_{0}}-\frac{\rho-\rho_{0}}{\rho_{0} c}
$$


Where $V$ is the volume, $n$ the number of solute molecules in moles, and $M$ is the molar mass of the solute. The very right term is valid only for diluted lipid suspensions as used in this study.

The adiabatic compressibility coefficient, $\beta_{S}=-1 / V(\partial V / \partial p)_{S}(V, p$ and $S$ are the volume, the pressure and entropy, respectively), the speed of sound propagation, $u$, in the medium, and the density, $\rho$ are related by the expression:

$$
\beta_{S}=1 / u^{2} \rho
$$

In molecular acoustics, due to the additivity of all components of the system, the partial molar adiabatic compressibility, $K_{S}^{\mathrm{o}}$, is generally used, which is given by;

$$
K_{S}^{\mathrm{o}}=\left(\frac{\partial K_{S}}{\partial n}\right)=\left(\frac{\partial V^{\mathrm{o}}}{\partial p}\right)_{S} \cong \beta_{S, 0}\left(2\left(V^{\mathrm{o}}-[u]\right)-\frac{M}{\rho_{0}}\right),
$$

where $K_{S}=\beta_{S} V$ is the adiabatic compressibility and $\beta_{S, 0}$ is the adiabatic compressibility coefficient of the solvent. By dividing the partial molar quantities $V^{\mathrm{o}}$ and $K_{S}^{\mathrm{o}}$ by the molar mass of the solute we obtain the partial specific values, i.e., the partial specific volume, $v^{\circ}$, and the partial specific adiabatic compressibility, $k_{S}{ }^{\circ}$. Accordingly, the concentration, $c$, in Eq. 1 becomes $c / M$, which is then expressed in $\mathrm{mg} / \mathrm{mL}$.

The sound velocity was determined with a relative error better than $10^{-3} \%$, corresponding to a precision higher than $5 \times 10^{-5} \mathrm{~mL} / \mathrm{g}$ in $[u]$. The density values were measured with relative error smaller than $10^{-3} \%$, so the accuracy in $v^{\circ}$ is better than $10^{-4} \mathrm{~mL} / \mathrm{g}$. Therefore, considering the relative errors of $[u]$ and $v^{0}$, the certainty in $k_{S}{ }^{\circ}$ taken from Eq. 4 is within $10^{-12} \mathrm{~mL} / \mathrm{gPa}$. In both methods, the corresponding values were measured at discrete temperatures (read with an accuracy of $10^{-3}{ }^{\circ} \mathrm{C}$ ), resulting in an average temperature scan rate of $\sim 12^{\circ} \mathrm{C} / \mathrm{h}$.

The adiabatic compressibility of the lipids, $\beta_{S}^{\text {lipid }}$, is defined as

$$
\beta_{S}^{\text {lipid }}=-\frac{1}{v^{\circ}}\left(\frac{\partial v^{\circ}}{\partial p}\right)_{S}
$$

which is related to the partial specific adiabatic compressibility, $k_{S}{ }^{\circ}$, by

$$
k_{S}^{\mathrm{o}}=v^{\mathrm{o}} \beta_{S}^{\text {lipid }}
$$

$\beta_{S}^{\text {lipid }}$ can thus be directly obtained from combined ultrasound velocity and density measurements.

Lipid bilayer thermotropic main phase transitions are considered to be of weak first-order, i.e., they show typical features of first-order phase transitions, such as abrupt changes in specific volume or a peak in the enthalpy and entropy, but also significant fluctuations in volume and lamellar $d$-spacing, which are typical for a second-order phase transition. The isothermal compressibility, $K_{T}$, is directly proportional to the volume fluctuations of the system [Wilson 1957, Hill 1960]. In a system exhibiting a first-order transition, $K_{T}$ diverges at the phase transition temperature, whereas it exhibits a power-law behavior $\left(K_{T} \propto\left|T-T_{\mathrm{c}}\right|^{-\gamma}\right)$, with a particular critical exponent $(\gamma=1.24$ for 3D systems) in the critical-point region of a second-order phase transition [Stanley 1971, Winter 1999]. By the ultrasound velocity and the density measurements, however, only the adiabatic compressibility, $K_{S}$, can be determined (see Eqn. 3 and 4). The isothermal compressibility can be calculated from [Hill 1960]

$$
K_{T}=K_{S} \frac{C_{p}}{C_{V}}
$$

where $C_{p}$ and $C_{V}$ are the heat capacities at constant pressure and volume, respectively, which, using Maxwell relations, can also be expressed as

$$
K_{T}=K_{S}+\frac{T}{C_{p}}\left(\frac{\partial V}{\partial T}\right)_{p}^{2}=K_{S}+\frac{T E^{2}}{C_{p}}
$$

with the thermal expansion $E=(\partial V / \partial T)_{p}$. Hence, the isothermal compressibility can be obtained from the adiabatic one when the thermal expansion and the heat capacity data are available. Differentiating Eqn. 8 yields the exact differential of $K_{T}, \mathrm{~d} K_{T}$, which is given as: 


$$
\mathrm{d} K_{T}=\mathrm{d} K_{S}+\frac{E^{2}}{C_{p}} \mathrm{~d} T+2 \frac{T E}{C_{p}} \mathrm{~d} E-\frac{T E^{2}}{C_{p}^{2}} \mathrm{~d} C_{p}
$$

For convenience, Eqn. 8 is adapted through Eqn. 9 by thermodynamic treatment to a form where the corresponding partial specific quantities are taken [Chalikian 2003]:

$$
k_{T}^{\mathrm{o}}=k_{S}^{\mathrm{o}}+\frac{T \alpha_{0}^{2}}{\rho_{0} c_{p, 0}}\left(2 \frac{e^{\mathrm{o}}}{\alpha_{0}}-\frac{C_{p}^{\mathrm{o}}}{\rho_{0} c_{p, 0}}\right)
$$

$k_{T}{ }^{\circ}$ is the partial specific isothermal compressibility, $\alpha_{0}(\alpha=E / V)$ and $c_{p, 0}$ are the thermal expansion coefficient and the specific heat capacity of the solvent, respectively; $e^{\mathrm{o}}$ and $C_{p}{ }^{\mathrm{o}}$ are the partial specific expansivity and the partial specific heat capacity of the lipid, respectively; the latter is given by [Privalov 1980]

$$
C_{p}^{\mathrm{o}}=\frac{\Delta C_{p}}{m}+\frac{v^{\mathrm{o}}}{v_{0}^{\mathrm{o}}} c_{p, 0}
$$

where $m$ is the mass of the solute.

The corresponding isothermal compressibility of the lipid, $\beta_{T}^{\text {lipid }}=k_{T}^{\mathrm{o}} / v^{\mathrm{o}}$ (note that $\beta_{T}^{\text {lipid }}$ differs from the partial specific isothermal compressibility coefficient, $\beta_{T}{ }^{\circ}$, which is defined as $\left.\beta_{T}{ }^{\circ}=1 / M\left(\partial \beta_{T} / \partial n\right)=\beta_{T} v^{\mathrm{o}} / V\right)$, can be obtained from Eqn. 10 and is given as:

$$
\beta_{T}^{\text {lipid }}=\beta_{S}^{\text {lipid }}+\frac{T \alpha_{0}^{2}}{v^{\mathrm{o}} \rho_{0} c_{p, 0}}\left(2 \frac{e^{\mathrm{o}}}{\alpha_{0}}-\frac{C_{p}^{\mathrm{o}}}{\rho_{0} c_{p, 0}}\right)
$$

For simplification, we denote in Eqn. 12 the second and third term as $\beta_{e}^{\text {lipid }}$, and $\beta_{C}{ }^{\text {lipid }}$, respectively:

$$
\beta_{T}^{\text {lipid }}=\beta_{S}^{\text {lipid }}+\beta_{e}^{\text {lipid }}-\beta_{C}^{\text {lipid }}
$$

Hence, the isothermal compressibility coefficient, $\beta_{T}^{\text {lipid }}$, is given as a sum of the adiabatic compressibility, $\beta_{S}^{\text {lipid }}$, an expansion term, $\beta_{e}^{\text {lipid, }}$, and a heat capacity term, $\beta_{C}$ lipid. Interestingly, as can be seen from Eqs. 10 and 12 , the heat capacity term has a compensating effect, balancing that of the thermal expansion on the adiabatic compressibility.

The thermodynamic parameters $C_{p}, K_{T}$ and $E$ are directly related to corresponding fluctuation parameters [Cooper 1984]: i) the square average of the enthalpy fluctuations, its variance, $\left\langle\Delta H^{2}\right\rangle$, is determined by the heat capacity, $C_{p}$, of the system, ii) the square average of the volume fluctuations $\left\langle\Delta V^{2}\right\rangle$ as given by the respective isothermal compressibility, $K_{T}$, and iii) the covariance between $H$ and $V,\langle\Delta H \Delta V\rangle$, is related to the thermal expansion, $E$ :

$$
\begin{gathered}
\left\langle\Delta H^{2}\right\rangle=R T^{2} C_{p} \\
\left\langle\Delta V^{2}\right\rangle=R T K_{T} \\
\langle\Delta H \Delta V\rangle=R T^{2} E=\mathrm{RT}^{2} V \alpha
\end{gathered}
$$

As seen from Eqn. 14c, the thermal expansion couples contributions from the heat capacity and the isothermal compressibility.

\section{Results and discussion}

\subsection{Ultrasound and density measurements on DPPC - gramicidin D $(g D)$ mixtures}

The determination of mechanical parameters of DPPC $-\mathrm{gD}$ is crucial for an evaluation of the size of distorted membrane structure around proteins or peptides (Hianik 1995). Figure 1a shows the temperature dependent velocity number $[u]$ for the DPPC - gramicidin mixtures. At points distant from the lipid main phase transition temperature, $T_{\mathrm{m}}$, [u], a gradual decrease of $[u]$ with rise in temperature is observed, leading to the typical anomalous dip (Mitaku 1978, Kharakoz 1993, Schrader 2002, Krivanek 2008) in the vicinity of $T_{\mathrm{m}}$ for pure lipids. The lowest value of $[u]$ at $T_{\mathrm{m}}$ is $\sim-0.15 \mathrm{~mL} / \mathrm{g}$ for pure DPPC, which is consistent with Mitaku and co-workers data (Mitaku 1978). It should be 
noted, however, that the size and the width of the dip in [u] depends on the sample preparation [Mitaku 1978], which is related to the different degree of cooperativity of the main phase transition, on the lipid concentration [Kharakoz 1993], and on the ultrasound frequency applied for the measurement itself [Mitaku 1982], which is related to the heat exchange within the period of the sound wave [Osdol 1989, Heimburg 1996]. The dip in the ultrasonic number profiles will be more pronounced at lower frequencies, because $C_{\mathrm{p}}$ assumes higher values, leading to an increase of the adiabatic compressibility at lower frequencies. The frequency dependence of the ultrasonic absorption coefficient of DPPC suspensions has been measured. The excess absorption data has been described by a relaxation term with a discrete relaxation time, displaying some evidence of critical slowing down near the phase transition [Heimburg 1996].

In addition, inadequately high temperature scan rates might induce a slight shift in the dip minimum position toward higher temperatures [Kaatze 2006], since lipid bilayers are not able to thermally equilibrate rapidly due to molecular processes slowing down during the main phase transition.

Addition of $1 \mathrm{~mol} \%$ gramicidin produced a significant change in $[u]$, and a peak broadening is observed leading to a comparatively increased $[\mathrm{u}]$ at $T_{\mathrm{m}}$ with a dip of $\sim-0.1 \mathrm{~mL} / \mathrm{g}$. The dip continues to decrease with increase in gramicidin concentration, with the highest value and broadest peak at $10 \mathrm{~mol} \%$. In the gel phase, in general, we observe that $[u]$ is smaller below $T_{\mathrm{m}}$ and larger above $T_{\mathrm{m}}$ for both DPPC - gramicidin mixtures than for the pure DPPC LUVs. In addition, for the DPPC - gramicidin mixtures in the gel phase, there is no clearly defined trend in $[u]$ unlike in the transition region. It is of interest to note that the minimum in the $[u]$ value generally reflects effects from changes in heat capacity, $C_{\mathrm{p}},[u]$ and the isothermal compressibility $\beta_{\mathrm{T}}$ upon approaching Tm.

The temperature dependence of the partial specific volume, $v^{0}$, is shown in figure $1 \mathrm{~b}$. An increase of $v^{0}$ with temperature is observed throughout the whole melting transition regime. A step-like change at the transition temperature $T_{\mathrm{m}}$ is observed for pure DPPC and for DPPC - gramicidin concentrations of 1 and $2.5 \mathrm{~mol} \%$. Changes in $[u]$ and $v^{0}$ with increase in gramicidin concentration are clearly observed at $T_{\mathrm{m}}$ and above the $T_{\mathrm{m}}$ region indicating, increased volume fluctuations in this temperature region. The $\Delta v^{\circ}$ for pure DPPC at the gel/fluid transition was found to be $\sim 0.045$, which corresponds to an $\sim 4 \%$ bilayer volume increase. The partial specific volume $v^{0}$ is larger below $T_{\mathrm{m}}$ and smaller above $T_{\mathrm{m}}$ for the DPPC - gramicidin mixtures, similar to the behavior of $[u]$.

The partial specific adiabatic compressibility, $k_{S}{ }^{\mathrm{o}}$, has been determined from equation 6 . Figure $2 \mathrm{a}$ gives the temperature dependence of $k_{S}{ }^{0}$ for the DPPC - gramicidin mixtures. As expected, the $k_{S}{ }^{0}$ for pure DPPC has the highest value increasing from $2.35 \mathrm{~mL} / \mathrm{gPa}$ at $10{ }^{\circ} \mathrm{C}$ and abruptly reaching $5.5 \mathrm{~mL} / \mathrm{gPa}(57 \%)$ at $T_{\mathrm{m}}$. It shows a slight drop right beyond $T_{\mathrm{m}}$, and finally continues to increase with increasing temperature to reach its maximum value of $6.3 \mathrm{~mL} / \mathrm{gPa}$ at $80^{\circ} \mathrm{C}$.

Generally, a significant broadening of the transition peak, a shift to lower temperatures and a decrease of $k_{S}{ }^{\circ}$ in the lipid melting transition region with increasing $\mathrm{gD}$ concentration was observed. Increase in compressibility of the lipid vesicle with increasing gramicidin concentration below $T_{\mathrm{m}}$ suggests a decrease in the lipid bilayer order. The opposite holds true for $T>T_{\mathrm{m}}$, where $k_{S}{ }^{\mathrm{o}}$ decreases with increasing gD concentration.

The temperature dependence of $\beta_{S}^{\text {lipid }}$ for the DPPC $-\mathrm{gD}$ mixtures is displayed in figure $2 \mathrm{~b}$. As can be seen it has the same shape as $k_{S}{ }^{\circ}$ (Eqn. 10), which includes the anomalous peak at the main phase transition.

At 25 and $60{ }^{\circ} \mathrm{C}, \beta_{S}^{\text {lipid }}$ for pure DPPC is $3.2 \times 10^{-10} \mathrm{~Pa}^{-1}$ and $5.6 \times 10^{-10} \mathrm{~Pa}^{-1}$, respectively. The value for $\beta_{S}^{\text {lipid }}$ of $3.4 \times 10^{-10} \mathrm{~Pa}^{-1}$ at $30^{\circ} \mathrm{C}$ is in good agreement with $\beta_{S}^{\text {lipid }}=3.5 \times 10^{-10} \mathrm{~Pa}^{-1}$ obtained by Mitaku and coworkers (Mitaku 1978), but $5.3 \times 10^{-10} \mathrm{~Pa}^{-1}$ at $50{ }^{\circ} \mathrm{C}$ is higher compared to the literature value of $4.6 \times 10^{-10} \mathrm{~Pa}^{-1}$, which might be due to different vesicle preparations (LUV in our study and MLV in the reference mentioned (Mitaku 1978)). Also for $k_{S}{ }^{\circ}$, the anomalous increase of $\beta_{S}^{\text {lipid }}$ around $T_{\mathrm{m}}$ is still significant at 1 and $2.5 \mathrm{~mol} \%$ of $\mathrm{gD}$ and markedly diminishes for higher $\mathrm{gD}$ concentrations.

A summary of the effects of increasing gramicidin concentration on $v^{\circ}$ and $\beta_{S}^{\text {lipid }}$ of the bilayer at 25 and $60^{\circ} \mathrm{C}$ is shown in figures $3 \mathrm{a} \& 3 \mathrm{~b}$, respectively. The data reveal a continuous decrease in $v^{\circ}$ and $\beta_{S}^{\text {lipid }}$ with $\mathrm{gD}$ concentration in the fluid phase (except between 5 and $7.5 \%$, where we observe only a very slight decrease) and a slight increase with concentration in the gel phase. The increase of $\beta_{S}^{\text {lipid }}$ with increasing gD concentration, in the gel phase probably reflects the disordering effect which $\mathrm{gD}$ imposes on ordered phospholipid bilayers in this phase region. The data thus clearly show that incorporation of $\mathrm{gD}$ also drastically changes the temperature-dependent gel phase behavior of DPPC. It has been reported that incorporation of gD leads to a decrease of the molecular order of the acyl chains in the gel phases, whereas in the fluid phase, the mean order parameter increases (Eisenblätter 2006). Incorporation of $\mathrm{gD}$ into fluid-like DPPC has been shown to have a significant rigidifying effect on the conformational order in a cooperative manner along the entire acyl chain. Furthermore, it can be seen that, as 
expected, both $v^{\mathrm{o}}$ and $\beta_{S}^{\text {lipid }}$ generally increase with increasing temperature, and the slope of $\beta_{S}^{\text {lipid }}(T)$ decreases with increase in $\mathrm{gD}$ concentration.

\subsection{Isothermal Compessibility and Volume fluctuations of DPPC $-g D$ mixtures}

Figure 4a displays the temperature dependences of the isothermal compressibility of DPPC-gD LUVs at different gD concentrations. We observed that the isothermal compressibility peak at the main transition drops drastically $(\sim$ $75 \%$ ) upon addition of gramicidin concentrations as low as $1 \mathrm{~mol} \%$. This decrease in $\beta_{T}^{\text {lipid }}$ corresponds to a similar strong decrease $(80 \%)$ of the thermal expansion coefficient, indicating the close relationship between the corresponding fluctuations $\left(\left\langle\Delta V^{2}\right\rangle v s .\langle\Delta H \Delta V\rangle\right)$ (Krivanek 2008).

Between 1 and $10 \mathrm{~mol} \% \mathrm{gD}$, only a slight decrease in $\beta_{T}^{\text {lipid }}$ at the main transition region with increasing $\mathrm{gD}$ concentration is observed, and almost no change between 5 and $7.5 \mathrm{~mol} \%$, as already observed in $\beta_{S}^{\text {lipid }}$. At gD concentrations higher than $5 \mathrm{~mol} \%, \beta_{T}^{\text {lipid }}$ is greater than $\beta_{S}{ }^{\text {lipid }}$ at the gel-fluid region by $\sim 10-20 \%$ in the whole temperature range covered. Significant differences in $\beta_{T}^{\text {lipid }}$ and $\beta_{S}^{\text {lipid }}$ are seen in the gel and fluid phases of the lipid bilayer, as the solvent and lipid membranes are adiabatically uncoupled in the $\mathrm{MHz}$ region in the ultrasound experiment. These differences become dramatic in the gel-fluid transition region, indicating a significant degree of slow relaxational processes in the $\mathrm{s}$ time range in the transition region. $\beta_{T}^{\text {lipid }}$ in both the gel phase $\left(25^{\circ} \mathrm{C}\right)$ and in the fluid phase $\left(60^{\circ} \mathrm{C}\right)$ of the DPPC-gD mixtures between 1 and $10 \mathrm{~mol} \%$ reveal continuous increase with increase in $\mathrm{gD}$ concentration (Fig. 5a). Above the main transition, at $60{ }^{\circ} \mathrm{C}$, only the $\beta_{T}^{\text {lipid }}$ value of $10 \mathrm{~mol} \%$ gramicidin surpasses the value for pure DPPC.

Given that the partial specific volume, vo, is largely determined by the lipid term (Eqn. 2), which reflects the "real" volume of the lipid molecule, Eqn. 3 can be modified to convey the relative volume fluctuations as

$$
\sqrt{\frac{\left\langle\Delta V^{2}\right\rangle}{V^{2}}}=\sqrt{\frac{R T \beta_{T}^{\text {lipid }}}{M v^{o}}}
$$

Fig. $4 \mathrm{~b}$ displays the calculated temperature dependence of the relative volume fluctuations for the DPPC-gD mixtures. The figure clearly shows that the relative volume fluctuations of pure DPPC are drastically increased at the main transition, reaching up to $12 \%$, and are strongly damped upon addition of gramicidin $\mathrm{D}$ to $2.6 \%$ at 10 $\mathrm{mol} \% \mathrm{gD}$. Furthermore, the volume fluctuations in both the gel phase $\left(25^{\circ} \mathrm{C}\right)$ and in the fluid phase $\left(60{ }^{\circ} \mathrm{C}\right)$ reveal a continuous and gradual increase with increase in $\mathrm{gD}$ concentration between 1 and $10 \mathrm{~mol} \%$ (Fig. 5b). Again, it can be clearly seen that the volume fluctuations are larger in the fluid phase than in the gel phase.

\subsection{Conclusion}

In this work, the isothermal compressibility and the volume fluctuations of DPPC - gramicidin bilayer membranes in their different phases was determined by using molecular acoustics (ultrasound velocity and densitometry). The effect of increasing gramicidin concentration on $v^{\circ}$ and $\beta_{S}^{\text {lipid }}$ of the bilayer at $T_{\mathrm{m}}$ region has revealed a continuous decrease in $v^{\circ}$ and $\beta_{S}^{\text {lipid }}$ (within the range of concentrations studied) with increase in $\mathrm{gD}$ concentration except between 5 and $7.5 \%$, where only a very slight decrease was observed. Another observation was that the isothermal compressibility peak at the main transition drops drastically $(\sim 75 \%)$ upon addition of gramicidin concentrations as low as $1 \mathrm{~mol} \%$. In addition, at $\mathrm{gD}$ concentrations higher than $5 \mathrm{~mol} \%$, it was found that $\beta_{T}^{\text {lipid }}$ is greater than $\beta_{S}^{\text {lipid }}$ in the gel-fluid coexistence region by $10-20 \%$ in the whole temperature range covered.

The maximum value of the relative volume fluctuation of $12 \%$ is reached for pure DPPC at the main transition, and is strongly damped upon addition of $\mathrm{gD}$. A gradual decrease in the calculated relative volume fluctuations with $\mathrm{gD}$ concentration at $T_{\mathrm{m}}$ is observed between concentrations 1 and $10 \mathrm{~mol} \%$.

\section{Acknowledgement}

Profound grateful goes to Prof. Dr. R. Winter, Department of Physical Chemistry, Dortmund University of Technology, Germany, in whose laboratory this work was carried out. Financial support from the Deutsche Forschungsgemeinschaft (DFG), Germany and the regional county of Northrhine Westfalia is gratefully acknowledged.

\section{References}

Bourinbaiar, A. S., \& C. F. Coleman. (1997). The effect of gramicidin, a topical contraceptive and antimicrobial agent with anti-HIV activity, against herpes simplex viruses type 1 and 2 in vitro. Arch Virol, 142, 2225-2235.

Burkhart, B. M. (1999). Gramicidin D conformation, dynamics and membrane ion transport. Biopolymers, 51, 129-144. 
Cevc, G., \& Marsh D. (1987). Phospholipid Bilayers, John Wiley and Sons, New York.

Chalikian, T. V. (2003). Volumetric Properties of Proteins. Annu. Rev. Biophys. Biomol. Struct, 32, 207-235.

Cooper, A., (1984). Protein fluctuations and the thermodynamic uncertainty principle. Prog. Biophys. Molec. Biol., 44, 181-214.

Eggers, F., \& Kustin K. (1969). Ultrasonic methods. Methods Enzymol, 16, 55-80.

Eggers, F., \& Funk T. (1973). Ultrasonic measurements with millilitre liquid sample in the $0.5-100 \mathrm{MHz}$ range. Rev. Sci. Instr, 44, 969-978.

Eisenblätter, J., \& Winter R. (2006). Pressure effects on the structure and phase behaviour of DMPC-Gramicidin lipid bilayers: A synchrotron SAXS and ${ }^{2} \mathrm{H}-\mathrm{Nmr}$ spectroscopy study. Biophys. J., 90, 956-966.

Ge, M. T., \& Freed J.H. (1999). Electron spin resonance study of aggregation of gramicidin in dipalmitoylphosphatidylcholine bilayers and hydrophobic mismatch. Biophys. J., 76, 264-280.

Glowka, M. L., Olzzak, A. Bojarska, J., Szczesio, M., Duax, W.L., Burkhart, B.M., Pangborn, W.A., Langs, D.A., \& Wawrzak, Z. (2005). Structure of gramicidin D-Rbcl complex at atomic resolution from low-temperature synchrotron data: interactions of double-stranded gramicidin channel contents and cations with channel wall. Biological crystallography, 61, 433-441.

Heimburg, T. \& Marsh D. (1996). Thermodynamics of the interaction of proteins with lipid membranes. In Biological Membranes: A Molecular Perspective from Computation and Experiment. K. M. Merz, and B. Roux. editors. Birkhauser, Boston. 405-462.

Hianik, T. \& Passechnik V.I. (1995). Bilayer Lipid Membranes: Structure and Mechanical Properties, Kluwer Academic, Dordrecht/ Boston/London.

Hill, T. L. (1960). An Introduction to Statistical Thermodynamics. Dover, New York.

Ivanova, V. P., Makarou, I.M., Schäffer, T.E., Heimburg T. (2003). Analysis, heat capacity profile of peptide containing membranes cluster formation of gramicidin A. Biophys. J., 84, 2427-2439.

Kaatze, U., O'Driscoll, B., Hanke, E., Jäger, M \& Buckin V. (2006). Ultrasonic calorimetry of membranes. Pharmaceutical Technology Europe, 1-5.

Kharakoz, D. P., Colotto, A., Loher, K., \& Laggner, P. (1993). Fluid-gel interphase line tension and density fluctuations in dipalmitoylphosphatidylcholine multilamellar vesicles: an ultrasonic study. J. Phys. Chem., 97, 9844-9851.

Killian, J. A. (1992). Gramicidin and gramicidin-lipid interactions. Biochim Biophys. Acta, 1113, 391-425.

Kratky, O., Leopold H., \& Stabinger H. (1973). The determination of partial specific volume of protein by the mechanical oscillator technique. Methods Enzymol, 27, 98-110.

Krivanek, R., Rybar P., Prenner, E.J \& McElhaney R.E. (2001). Interaction of the antimicrobial peptide gramicidin $\mathrm{S}$ with dimyristoyl-phoshatidyl choline bilayer membranes: A densitometry and sound velocimetry study Biochim. Biophys. Acta, 1510, 452-463.

Krivanek, R., Okoro, L., \& Winter, R. (2008). Effect of cholesterol and ergosterol on the compressibility and volume fluctuations of phospholipid-sterol bilayers in the critical point region - A molecular acoustic and calorimetric study. Biophys. J., 94, 3538-3548.

Lewis, R. N. A. H., Prenner, E.J., Kondejewski, L.H., Flash C.R., Mendelsohn, R., Hodges, R.S., \& McElhaney, R.N. (1999). Fourier transform infrared spectroscopic studies of the interaction of Antimicrobial peptide Gramicidin $\mathrm{S}$ with micelles and with lipid monolayer and bilayer membranes. Biochemistry. 38, 15193-15203.

MacDonald, R. C., MacDonald, R.I., Menco, B.P.M., Takeshita, K., Subbarao, N.K., \& Hu, L.R. (1991). Small-volume extrusion apparatus for preparation of large unilamellar vesicles. Biochim. Biophys. Acta. 1061, 297-303.

Mitaku, S., Ikegami, A \& Sakanishi, A. (1978). Ultrasonic studies of lipid bilayer phase transition in synthetic phosphatidylcholine liposomes. Biophys. Chem, 8, 295-304.

Mitaku, S., \& Data, T. (1982). Anomalies of nanosecond ultrasonic relaxation in the lipid bilayer transition. Biophys. Biochim. Acta, 688, 411-421.

Orädd, G., \& Lindblom, G. (2004). Nmr studies of lipid lateral diffusion in the DMPC/gramicidin D/water system: peptide aggregation and obstruction effects. Biophys. J., 87, 980-987. 
Okoro, L., \& Winter, R. (2008). Pressure Perturbation Calorimetric Studies on Phospholipid-Sterol Mixtures. Z. Naturforsch, 63b, 769-778.

Osdol, V. W. W., Biltonen, R.L., \& Johnson, M.L. (1989). Measuring the kinetics of membrane phase transition. J. Bioenerg. Biophys. Methods, 20, 1-46.

Privalov, P. L. (1980). Scanning microcalorimeters for studying macromolecules. Pure Appl. Chem., 52, 479-497.

Rice, D. \& Oldfield, E. (1979). Deuterium nuclear magnetic resonance studies of the interaction between dimyristoylphosphatidylcholine and gramicidin A. Biochemistry, 18, 3272-3279.

Schrader, W., Ebel, H., Grabitz, P., Hanke, E., Heimburg, T., Hoeckel, M., Kahle, M., Wente, F., \& Kaatze, U. (2002). Compressibility of Lipid mixtures studied by calorimetry and ultrasonic velocity measurements. J. Phys. Chem, 106, 6581-6586.

Short, K. W., Wallace, B.A., Myers, R.A., Fodor, S.P.A., \& Dunker, A.K. (1987). Comparison of lipid/gramicidin dispersion and co-crystals by Roman scattering. Biochemistry, 26, 557-562.

Stanley, H. E. (1971). Introduction to Phase Transitions and Critical Phenomena. Oxford University Press, NY.

Stuehr, J., \& Yeager, E. (1965). The Propagation of Sound in Electrolytic Solutions. In Physical Acoustics. Vol. 2A. W. P. Mason. editor. Academic Press, NY.

Szule, J. A., \& Rand, R.P. (2003). The effects of gramicidin on the structure of phospholipid assemblies. Biophys. J., $85,1702-1712$.

Troiano, G. C., Stebe, K.J., Raphael, R.M., \& Tung, L. (1999). The effects of gramicidin on electroporation of lipid bilayers. Biophys. J., 76, 3150-3157.

Wilson, A. H. (1957). Thermodynamics and statistical mechanics. Cambridge University Press, Cambridge.

Winter, R., Gabke, A., Czeslik,C., \& Pfeifer, P. (1999). Power-law fluctuations in phase-separated lipid membranes. Phys. Rev., E. 60, 7354-7359.

Woolf, T. B. \& Roux, B. (1994). Molecular dynamic simulations of the gramicidin channel in a phospholipid bilayer. Proc. Natl. Acad. Sci., USA. 91, 11631-11635.

Woolf, T. B. \& Roux, B. (1996). Structure, energetics and dynamics of lipid-protein interactions: a molecular dynamics study of the gramicidin A channel in a DMPC bilayer. Proteins, 24, 92-114.

Zein, M. \& Winter, R. (2000). Effect of temperature, pressure and lipid acyl-chain length on the structure and phase behaviour of phospholipid-gramicidin Bilayers", Phys. Chem. Chem. Phys., 2, 4545-4551.

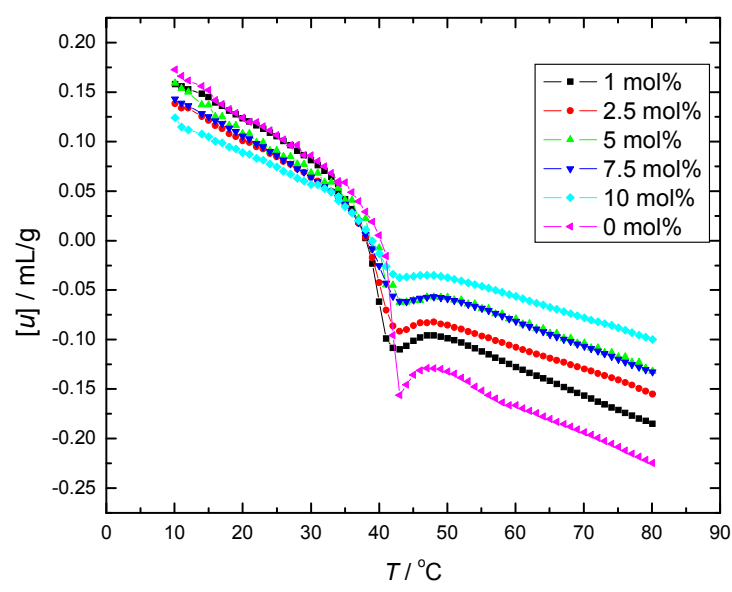

a

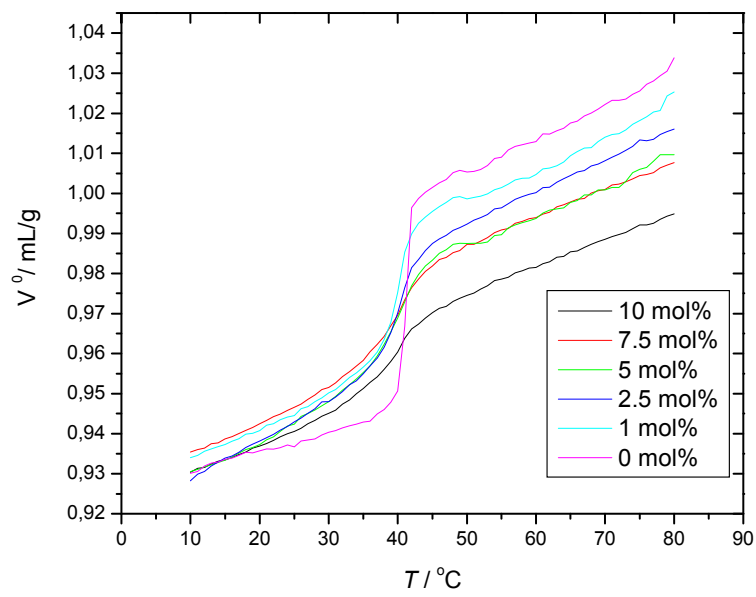

b

Figure 1. a) The temperature dependence of the ultrasound velocity number, $[u]$, for DPPC $-\mathrm{gD}$ mixtures. b) The temperature dependence of the partial specific volume, $v$, for DPPC $-\mathrm{gD}$ mixtures 
a)

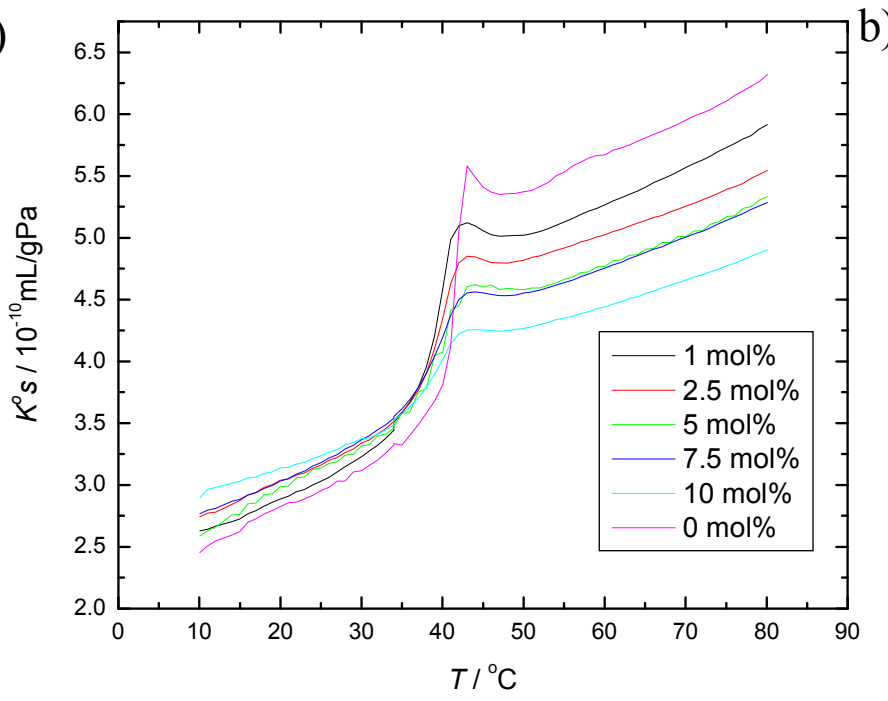

b)

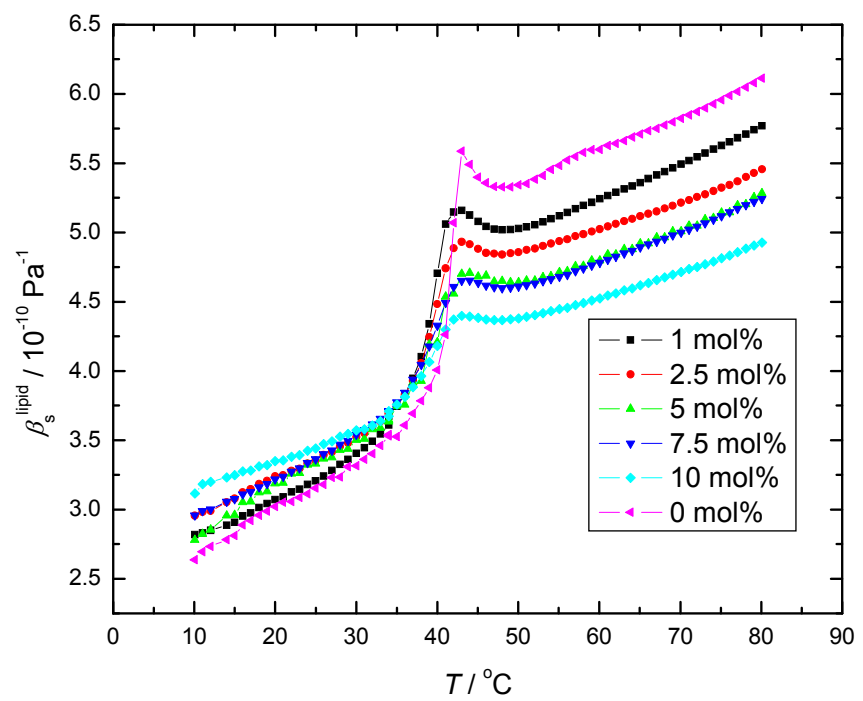

Figure 2. a) The temperature dependence of the partial specific adiabatic compressibility, $k_{S}{ }^{\circ}$, of DPPC-gramicidin mixtures at different gramicidin mole fractions, $X_{\mathrm{gD}}$ and (b) the adiabatic compressibility coefficient, $\beta_{S}^{\text {lipid, }}$, of DPPC LUVs at various gramicidin mole fractions

a)

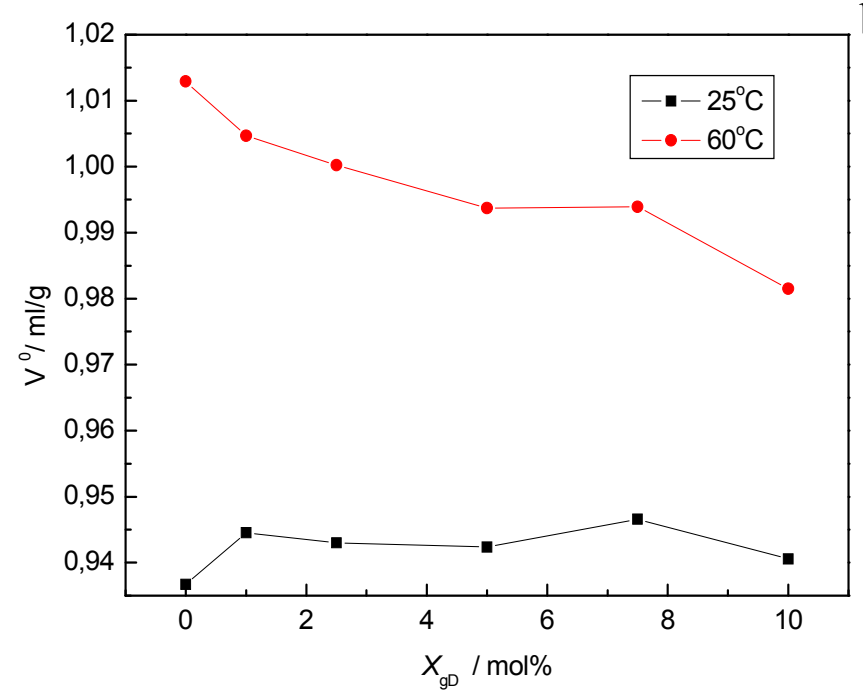

b)

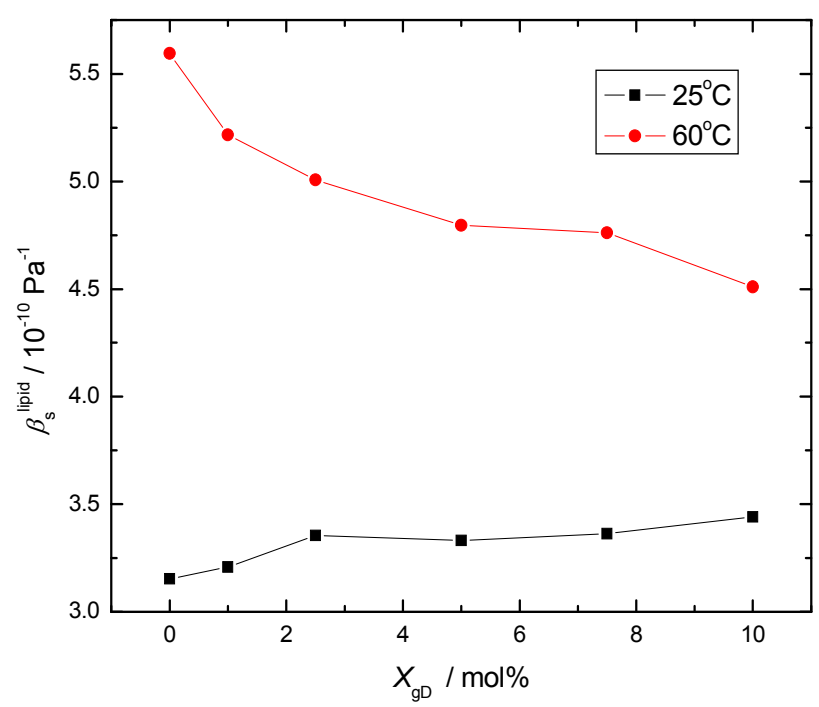

Figure 3. a) The partial specific volume and (b) the adiabatic compressibility coefficient of DPPC-gD mixtures as a function of $\mathrm{gD}$ concentration at $25^{\circ} \mathrm{C}$ and $60^{\circ} \mathrm{C}$. 
a)

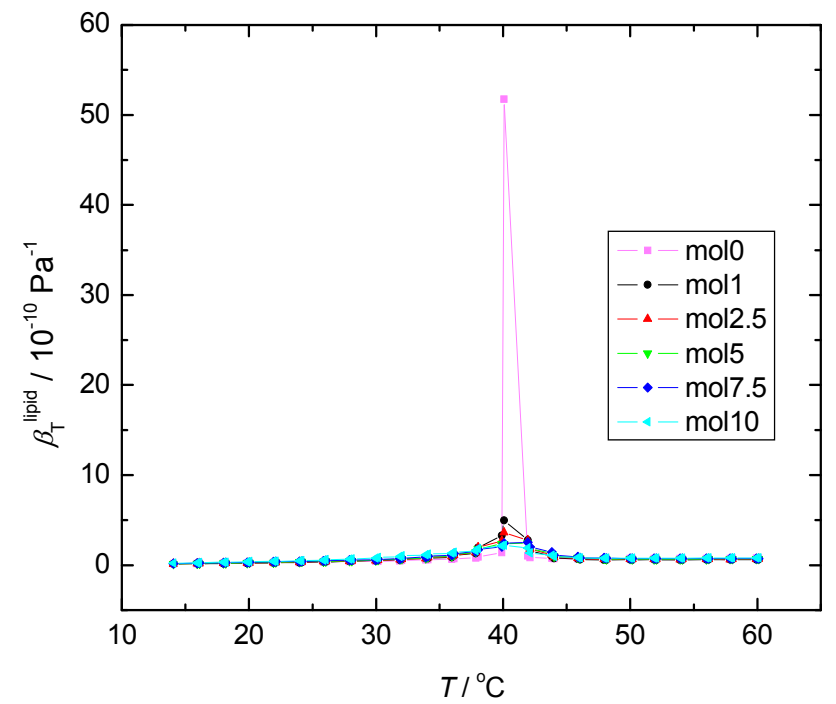

b)

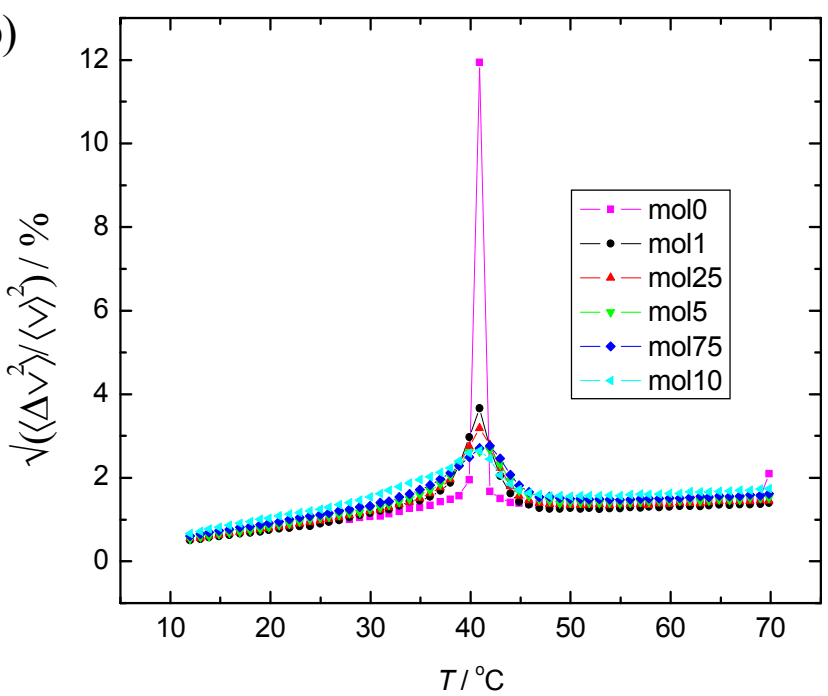

Figure 4. The temperature dependence of (a) the isothermal compressibility coefficient of the lipids $\beta_{T}^{\text {lipid }}$ and (b) the calculated relative volume fluctuations for DPPC-gD mixtures at different $\mathrm{gD}$ molar fractions, $X_{g D}$
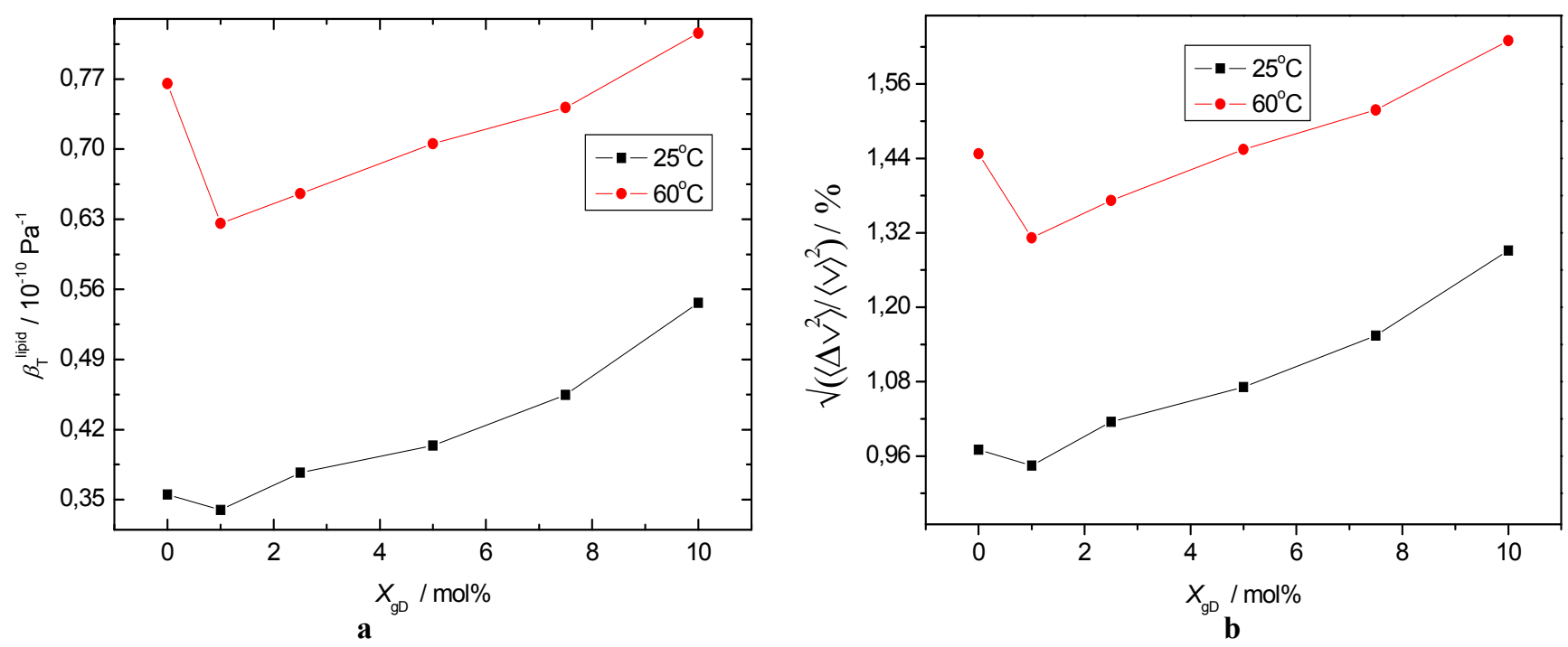

Figure 5. a) The temperature dependence of the calculated isothermal compressibility coefficient of the lipids, $\beta_{T}^{\text {lipid }}$, at 25 and $60{ }^{\circ} \mathrm{C}$ for DPPC-gD mixtures at different $\mathrm{gD}$ mole fractions, $\mathrm{X}_{g D}$. (b) the temperature dependence of the calculated relative volume fluctuations at $25^{\circ} \mathrm{C}$ and $60^{\circ} \mathrm{C}$ for DPPC-gD mixtures at different $\mathrm{gD}$ mole fractions, $\mathrm{X}_{g D}$ 